\title{
Efficacité comparée pour la croissance du poussin de différentes sources de méthionine
}

\author{
DC Bruyer *, M Vanbelle \\ Université catholique de Louvain, laboratoire de biochimie de la nutrition, \\ B-1348 Louvain-la-Neuve, Belgique
}

(Reçu le 30 mai 1989 ; accepté le 4 janvier 1990)

\begin{abstract}
Résumé - Nous avons comparé les aptitudes de la DL-méthionine, de la méthionine hydroxy analogue et de la forme dimérique de la méthionine hydroxy analogue à valoriser les protides d'une ration formulée au moyen de maīs et de farine de soja. L'efficacité des sources de méthionine est appréciée à partir de la croissance et des indices de consommation, contrôlés pendant 2 semaines.

Un aliment de base (3200 kcal $/ \mathrm{kg}, 21,65 \%$ de matières azotées totales) déficient en acides aminés soufrés $(0,65 \%$ méthionine + cystine) est supplémenté d'une dose unique d'un des 3 produits testés $(0,2 \%)$ équivalente à une addition équimolaire de DL-méthionine. L'addition est effectuée aux dépens du maìs concassé. Les aliments expérimentaux sont distribués ad libitum à des poussins mâles «Hubbard" du jour 7 au jour 19.

Dans ces conditions, la méthionine hydroxy analogue et sa forme dimérique possèdent la même efficacité que la DL-méthionine lorsque ces substances sont comparées sur base du produit actif. On observe la même croissance pondérale, la même consommation d'aliment et la même efficacité des régimes.
\end{abstract}

poulet / DL-méthionine / DL-méthionine hydroxy analogue / DL-méthlonine hydroxy analogue dimère

Summary - Efficiency of methionine sources for chicken growth. The present experiment was carried out to study the ability of DL-methionine, methionine hydroxy analogue and its dimeric form to improve the protein value of a maize-soybean meal poultry diet. Efficiency of methionine sources was appreciated for 2 weeks from growth and feed conversion ratio.

A basal diet ( $3200 \mathrm{kcal} / \mathrm{kg}, 21.65 \%$ crude protein) deficient in sulfur amino acids $(0.65 \%$ methionine + cystine) was supplemented with a single dose of the 3 tested substances at the level of $0.2 \%$, equivalent to DL-methionine on molar basis, in place of maize. The experimental diets were fed ad libitum to male Hubbard broilers from day 7 to day 19. Under these conditions, methionine hydroxy analogue and its dimeric form exhibited, on molar basis, a similar efficiency to DL-methionine. This experiment confirms the bioavailability of HMB dimer for chicks. Results obtained during previous in vitro studies are not in conflict.

broller / DL-methlonine / DL-methionine hydroxy analogue / DL-methlonine hydroxy analogue dimer

\footnotetext{
* Correspondance et tirés à part
} 


\section{INTRODUCTION}

Dans les aliments usuels, la méthionine hydroxy analogue, acide DL-2-hydroxy-4(méthylthio) butanoïque (HMB) est une source de méthionine chez le poulet de chair et le dindon (Elkin et Hester, 1983 ; Noll et al, 1984 ; Garlich, 1985 ; Buresh et Harms, 1986 ; Bruyer et al, 1987 ; Bougon et al, 1988). Cette source de méthionine est disponible commercialement sous forme de produit liquide, solution aqueuse à $88 \%$ de méthionine hydroxy analogue. Comme d'autres acides hydroxycarboxyliques, le HMB polymérise en solution concentrée. Cette polymérisation fournit à l'équilibre $65 \% \mathrm{p} / \mathrm{p}$ de $\mathrm{HMB}, 20 \% \mathrm{p} / \mathrm{p}$ de HMB dimère et $3 \% p / p$ de HMB trimère ; elle résulte d'une estérification intermoléculaire réversible.

La valeur nutritionnelle de la fraction polymérisée est contestée dans plusieurs travaux (Boebel et Baker, 1982a ; Van Weerden et al, 1983 ; Kirchgessner et Steinhart, 1984 ; Muramatsu et al, 1984). La stabilité du HMB dimère est décrite par Koban et Koberstein (1984) en solution aqueuse et par Bruyer et al (1988) dans un milieu gastrique simulé $(\mathrm{pH} \mathrm{2,0}$; présence de pepsine). Néanmoins, dans un système in vitro simulant le milieu duodénal du poulet, Lawson et Ivey (1986) ont montré qu'une rapide hydrolyse enzymatique du HMB dimère avait lieu. Cinq des enzymes responsables de cette hydrolyse ont été identifiées au niveau intestinal (Bruyer et al, 1988).

Dans la présente étude, nous avons comparé l'efficacité du HMB dimère pour la croissance du poussin à celle de la méthionine hydroxy analogue et à celle de la DL-méthionine.

\section{MATÉRIEL ET MÉTHODES}

L'expérimentation a porté sur des poussins mâles "Hubbard" âgés d'un jour. Les coquelets sont nourris jusqu'à $7 \mathrm{j}$ d'un aliment équilibré du commerce. lls sont alors pesés individuellement et 80 d'entre eux sont répartis en 16 lots homogènes de 5 individus selon la méthode des blocs, d'après leur poids vif.

Quatre aliments sont préparés : un aliment de base et 3 aliments supplémentés. L'aliment de base (tableau la) est composé principalement de farine de soja et de maïs concassé. II contient $3200 \mathrm{kcal}$ d'énergie métabolisable (calculée) et $21,65 \%$ de matères azotées totales (analysées). Ces matières azotées sont déficientes en méthionine $(0,31 \%)$ et en acides aminés soufrés $(0,65 \%)$.

Tableau la. Composition centésimale et analyse chimique de l'aliment de base. (1): tableau lb; (2): $78 \%$ lysine.

Composants $\quad \%$

$\begin{array}{lr}\text { Maïs } & 58,0 \\ \text { Farine de soja 50 } & 34,0 \\ \text { Farine de poisson } & 1,0 \\ \text { Huile de soja } & 4,0 \\ \text { Minéraux + Vitamines (1) } & 1,0 \\ \text { Phosphate bicalcique } & 1,2 \\ \text { Carbonate de calcium } & 0,6 \\ \text { L-lysine HCl (2) } & 0,2\end{array}$

Composition chimique

Protéine brute $\quad 21,65$

Lysine $\quad 1,25$

Méthionine $\quad 0,31$

Méthionine + Cystine $\quad 0,65$

Calcium $\quad 0,90$

Phosphore disponible $\quad 0,45$

Énergie métabolisable calculée $(\mathrm{kcal} / \mathrm{kg})$ 
Tableau lb. Composition du mélange minéral vitaminé.

\begin{tabular}{|c|c|}
\hline Composants & /kg du régime \\
\hline \multicolumn{2}{|l|}{ en UI : } \\
\hline Vitamine A & 15000 \\
\hline Vitamine D3 & 2000 \\
\hline \multicolumn{2}{|l|}{ en mg : } \\
\hline Vitamine $\mathrm{E}$ & 15 \\
\hline Ménadione & 5 \\
\hline Vitamine C & 25 \\
\hline Thiamine & 1 \\
\hline Riboflavine & 6 \\
\hline Pyridoxine $\mathrm{HCl}$ & 2 \\
\hline Pantothénate $\mathrm{Ca}$ & 10 \\
\hline Acide nicotinique & 25 \\
\hline Vitamine $\mathrm{B} 12$ & 0,025 \\
\hline Acide folique & 0,5 \\
\hline Choline & 500 \\
\hline Biotine & 0,1 \\
\hline Sodium & 1100 \\
\hline Chlore & 1000 \\
\hline Manganèse & 70 \\
\hline Zinc & 80 \\
\hline Fer & 25 \\
\hline Cuivre & 12 \\
\hline Cobalt & 0,6 \\
\hline lode & 1,5 \\
\hline Sélénium & 0,1 \\
\hline Ethoxyquine & 60 \\
\hline Salinomycine & 60 \\
\hline Virginamycine & 15 \\
\hline
\end{tabular}

Dans le régime supplémenté en DLméthionine, l'addition de celle-ci $(0,2 \%)$ est effectuée pour ramener le niveau du facteur limitant du régime à celui recommandé par l'INRA (1984). Les supplémentations en dérivés hydroxylés des 2 autres régimes sont réalisées pour fournir, sur une base équimolaire, une quantité équivalente en méthionine. Toutes ces supplémentations (méthionine, méthionine hydroxy analogue, HMB dimère) sont effectuées aux dépens du maïs concassé.

Le HMB dimère a été produit par le laboratoire de chimie organique de l'Université Catholique de Louvain à Louvain-la-Neuve (Belgique). Sa préparation a été réalisée selon le procédé de Lawson et Ivey (1986). Sa pureté en HMB, mesurée par résonance magnétique nucléaire, était supérieure à $98 \% \mathrm{p} / \mathrm{p}$ et une teneur en monomère résiduel de $1 \% \mathrm{p} / \mathrm{p}$ était détectée par hplc.

Tous les aliments sont préparés et analysés avant le début de l'expérimentation. Les niveaux de supplémentation de chaque lot d'aliment sont vérifiés par hplc pour le dosage de la méthionine hydroxy analogue et du HMB dimère (Baudichau et al, 1987) ou par chromatographie liquide d'échange d'ions pour le dosage de la DLméthionine supplémentaire et des acides aminés de la protéine de l'aliment (Moore et al, 1958 ; Spackman et al, 1958).

Chacun des 4 régimes est distribué du j 7 au j 19 à 5 coquelets. Les animaux sont logés en batteries dans un local thermostaté et sous éclairage constant. L'aliment et l'eau sont distribués à volonté. A la fin de la période expérimentale, les gains de poids corporel sont mesurés individuellement après un jeûne alimentaire d'une durée de $10 \mathrm{~h}$; les consommations d'aliments sont déterminées et les indices de consommation calculés.

Les résultats de gain de poids, de consommation alimentaire et des indices de consommation ont été soumis à l'analyse statistique de la variance selon un modèle à un critère fixe (traitement) et les moyennes des traitements ont été comparées selon le test de Student Newman et Keuls.

\section{RÉSULTATS ET DISCUSSION}

Lorsque le HMB dimère est incorporé au maiis concassé d'abord, dans l'aliment expérimental ensuite, une hydrolyse importante du HMB dimère modifie le produit testé comme source de méthionine (tableau II). Le contenu en HMB monomère (exprimé en \% du total HMB monomère + $\mathrm{HMB}$ dimère) augmente d'une valeur de $1 \%$ p/p dans le produit à $23 \%$ dans l'aliment expérimental. Parallèlement, le HMB dimère, dosé à $99 \% \mathrm{p} / \mathrm{p}$ dans le produit testé, passe à $82 \% \mathrm{p} / \mathrm{p}$ lors du prémélange sur le maïs concassé (prémix à $5 \% p / p)$, puis se stabilise à $77 \% p / p$ dans l'aliment expérimental. Cette hydrolyse du 
Tableau II. Modification de l'équilibre monomère/dimère lors de la préparation des aliments expérimentaux (en \% du total monomère + dimère). (1) : monomère/dimère ; (2) : correspond au premier et au dernier jour de l'essai (jours 7 et 19 pour les poussins).

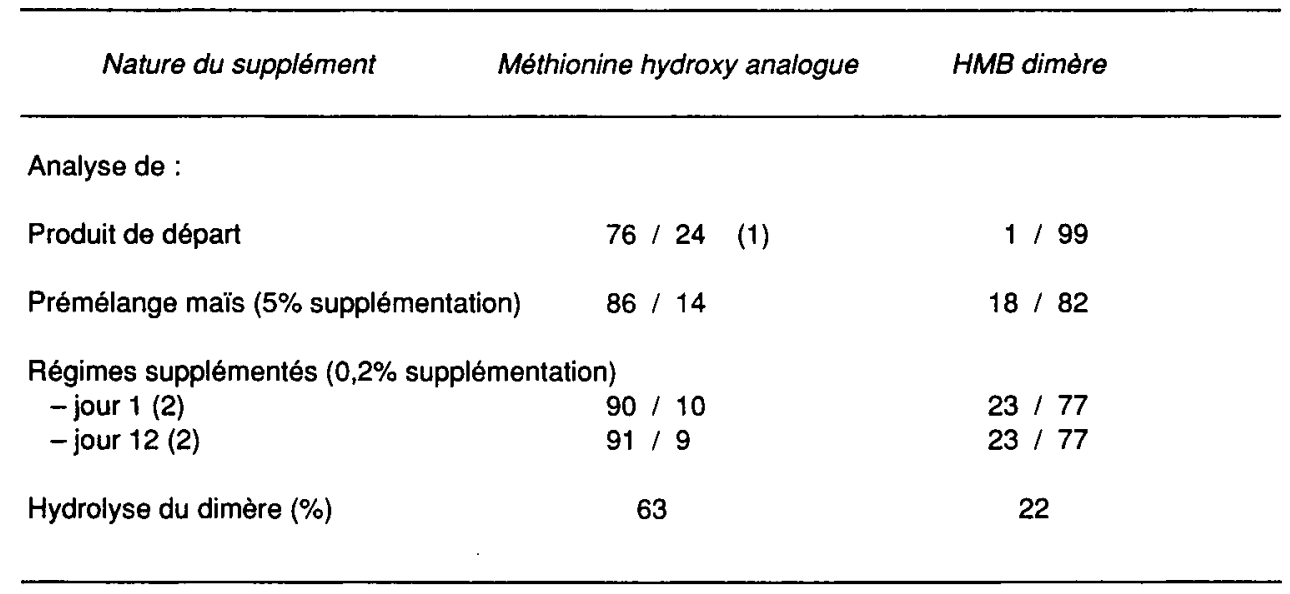

HMB dimère en HMB monomère est très rapide puisque les dosages ont été réalisés dans les minutes qui suivaient l'incorporation du HMB. En outre, une moindre extraction de la fraction polymérique durant la préparation de l'échantillon n'explique pas le contenu plus faible en HMB dimère puisque tout le HMB supplémenté est retrouvé dans l'extrait analysé.

Cette hydrolyse en monomère est également observée lors de la supplémentation de l'aliment par la préparation commerciale de méthionine hydroxy analogue qui contenait $23 \%$ d'oligomères et on peut raisonnablement estimer que plus de $60 \%$ du HMB dimère est hydrolysé lors de l'incorporation dans des aliments pour volaille (tableau II). La position exacte du nouvel équilibre est néanmoins affectée par la teneur en humidité de l'aliment, par la température dégagée lors de la mise en granulés et par le niveau de supplémentation en méthionine hydroxy analogue (données non publiées).
Ces résultats indiquent clairement que la concentration en oligomères de la méthionine hydroxy analogue mesurée avant l'incorporation à l'aliment aurait peu de signification pour le nutritionniste puisque l'équilibre sera modifié dans le sens d'un accroissement de la teneur en HMB monomère.

Les performances zootechniques moyennes des coquelets sont réunies dans le tableau III. Le poids moyen initial était de $138 \mathrm{~g}$ et le gain journalier de poids a été de 41,2 $\mathrm{g}$ par animal et par jour lors de la période expérimentale.

La carence en acides aminés soufrés du régime de base apparaît clairement puisque tous les lots d'animaux recevant une des sources de méthionine ont présenté un gain de poids significativement supérieur $(P<0,001)$ à celui observé avec les animaux sous-alimentés en méthionine. La réponse à la supplémentation a été de $8 \%$ pour le gain de poids. En revanche, la quantité d'aliment ingérée par 
Tableau III. Influence de la supplémentation du régime en source de méthionine sur les performances zootechniques des coquelets. (1) : moyenne de 4 répétitions de 5 coquelets pour la période $7-19 \mathrm{j}$ après l'éclosion ; poids moyen initial $138 \mathrm{~g}$; (2) : niveau de supplémentation $0,2 \%$ en méthionine, base équimolaire; (3) : non significatif.

Régimes

Gain de poids (g) Aliment ingéré (g)

Indice de consommation

\begin{tabular}{lccc} 
Aliment de base (B) & $470 \mathrm{a}$ & 791 & $1,69 \mathrm{a}$ \\
B + DL-méthionine (2) & $499 \mathrm{~b}$ & 763 & $1,53 \mathrm{~b}$ \\
B + Méthionine hydroxy analogue (2) & $508 \mathrm{~b}$ & 796 & $1,56 \mathrm{~b}$ \\
B + HMB dimère (2) & $499 \mathrm{~b}$ & 779 & $1,56 \mathrm{~b}$ \\
$\begin{array}{l}\text { Erreur standard de l'effet régime } \\
\quad \text { et niveau de signification }\end{array}$ & 4,99 & 20,87 & 0,033 \\
\hline
\end{tabular}

les coquelets n'a pas été modifiée par la supplémentation. Globalement, l'indice de consommation s'améliore significativement $(P<0,05)$ suite à l'apport de $0,2 \% \mathrm{p} / \mathrm{p}$ de sources de méthionine de synthèse. L'amélioration de l'indice de consommation a été de plus de $10 \%$.

La comparaison des traitements de supplémentation ne permet pas de détecter de différence significative entre la DLméthionine, la méthionine hydroxy analogue et le HMB dimère, et ce, tant pour les mesures de gain de poids que pour les indices de consommation.

Les performances observées avec le HMB dimère ont été similaires à celles obtenues avec la DL-méthionine ou la méthionine hydroxy analogue. Lors d'études antérieures concernant l'utilisation des polymères du HMB, Lawson et Ivey (1986) ont montré que $50 \%$ du HMB dimère étaient hydrolysés en monomère en moins de $\mathbf{3 0}$ min lors d'incubations in vitro dans une solution tamponnée contenant les enzymes de la pancréatine. De plus, l'addition de pancréatine fraîchement préparée permettait à l'hydrolyse de se poursuivre alors que l'utilisation d'un milieu d'incubation dénaturé par la chaleur ou d'un tampon sans enzymes empêchait toute hydrolyse du HMB dimère (Knight et Dibner, 1984). Ces résultats confirment que l'activité hydrolytique dérive d'une ou plusieurs enzymes d'origine pancréatique, solubles dans l'eau. Récemment, Bruyer et al (1988) ont pu montrer que plusieurs enzymes (l' $\alpha$-chymotrypsine, la $\beta$ chymotrypsine, la carboxypeptidase $A$, la carboxypeptidase B et la cholestérol estérase) sont responsables de l'hydrolyse rapide de HMB dimère au niveau intestinal.

D'autre part, l'incubation in vitro du HMB dimère dans une anse excisée d'intestin grêle pendant $2 \mathrm{~h}$ a montré que l'hydrolyse était alors pratiquement complète puisque le HMB monomérique a vu sa teneur passer de moins de $1 \%$ à $92 \%$ en fin d'incubation (Lawson et Ivey, 1986). Comme le temps de séjour minimal dans le tractus digestif d'un coquelet âgé de 10 à 14 j est compris entre 2 et $4 \mathrm{~h}$, l'exposition du HMB dimère aux enzymes intestinales ne semble pas être un facteur limitant. 
La potentialité du HMB dimère en tant que source de méthionine pour l'alimentation n'est pas une exception. Ainsi, le dipeptide DLLD méthionylméthionine permet-il de promouvoir la croissance des poulets de façon tout à fait équivalente à la DL-méthionine (Boebel et Baker, 1982b). En outre, ces peptides, par le fait qu'ils possèdent un groupement aminé protégé dans la liaison peptidique, présentent une efficacité améliorée, par rapport à la L-méthionine, après avoir subi un traitement thermique simulant les réactions de brunissement de Maillard (Baker et al, 1984).

\section{CONCLUSION}

Cette expérimentation confirme les potentialités de l'utilisation de la méthionine hydroxy analogue en tant que source de méthionine chez le poulet de chair. La présence de HMB dimère dans les préparations n'est pas limitante puisque la fraction dimérique est largement hydrolysée lors de la préparation de l'aliment et surtout parce que, in vivo, le poulet utilise cette molécule en tant que source effective de méthionine pour la croissance.

\section{RÉFÉRENCES}

Baker DH, Bafundo KW, Boebel KP, Czarnecki GL, Halpine KM (1984) Methionine peptides as potential feed supplements: efficacy and susceptibility to Maillard browning. J Nutr 114, 292-297

Baudichau A, Bruyer DC, Ontiveros R, Shermer WD (1987) A rapid hplc method for the determination of methionine hydroxy analogue free acid in supplemented feeds. I Sci Food Agric 38, 1-8
Boebel KP, Baker DH (1982a) Efficacy of the calcium salt and free acid forms of methionine hydroxy analog for chicks. Poult Sci 61, 1167-1175

Boebel KP, Baker DH (1982b) Efficacy of methionine peptides as determined by chicks bioassay. J Nutr 112, 1 130-1132

Bougon M, L'Hospitalier R, Launay M, Le Menec M (1988) Efficacité comparée de la méthionine et de la méthionine hydroxy analogue chez le poulet de chair. Bull Inf Stat Exp Avic Ploufragan 28

Bruyer DC, Baudichau A, Vanbelle M (1987) Méthionine en poudre ou liquide : même efficacité chez le poulet de chair. Aliscope 87(78), 28-30

Bruyer DC, Vanbelle M, Baudichau A (1988) Hydrolysis of 2-hydroxy-4-(methylthio) butanoic acid dimer in simulated intestinal fluid. Biotechnology and Food Industry. Proc Int Symp Budapest, 163-172

Buresh RE, Harms RH (1986) Comparison of DL-methionine and Aliment with broilers when full fed or restricted. Nutr Rep lnt 33 , 449-457

Elkin RG, Hester PY (1983) A comparison of methionine sources for broiler chickens fed corn-soybean meal diets under simulated commercial grown-out conditions. Poultry Sci $62,2030-2043$

Garlich JD (1985) Response of broiler to DLmethionine hydroxy analog free acid, DLmethionine and L-methionine. Poult Sci 64, 1541-1548

INRA (1984) L'Alimentation des animaux monogastriques. INRA éditeur, Paris, 282

Kirchgessner M, Steinhart H (1984) Biological effectiveness of DL-methionine-Na and DL-2hydroxy-4-methylthio butyric acid with broilers. Arch Geflugelk 48, 45-51

Knight CD, Dibner JJ (1984) Comparative absorption of 2-hydroxy-4-(methylthio) butanoïc acid and L-methionine in the broiler chick. $J$ Nutr 114, 2179-2186

Koban HG, Koberstein E (1984) Kinetics of hydrolysis of dimeric and trimeric methionine hydroxy analogue free acid under physiological conditions of $\mathrm{pH}$ and temperature. J Agric Food Chem 32, 393-396 
Lawson CQ, Ivey FJ (1986) Hydrolysis of 2hydroxy-4-(methylthio) butanoïc acid dimer in two model systems. Poult Sci 65, 1749 1753

Moore S, Spackman DH, Stein WH (1958) Chromatography of amino acids on sulfonated polystyrene resins. An improved system. Anal Chem 30, 1185-1 190

Muramatsu T, Yokota $\mathrm{H}$, Okumura J, Tasaki I (1984) Biological efficacy of liquid methionine and methionine hydroxy analogue free acid in chicks. Poult Sci 63, 1453-1 456
Noll SL, Waibel PE, Cook RD, Witmer JA (1984) Biopotency of methionine sources for young turkeys. Poult Sci 63, 2458-2470

Spackman DH, Stein WH, Moore S (1958) Automatic recording apparatus for use in the chromatography of amino acids. Anal Chem 30, 1190-1206

Van Weerden EJ, Schutte JB, Bertram HL (1983) DL-methionine and DL-methionine hydroxy analogue free acid in broiler diets. Poult Sci 62, 1269-1274 